Revista de Estudios Histórico-Jurídicos

[Sección historia del derecho patrio]

XLI (Valparaíso, Chile, 2019)

[pp. 305- 315]

\title{
Blackstone y SU USO POR BELLO EN LA FORMACIÓN DEL Código Civil de Chile. Un ejemplo en sede de nulidad DEL MATRIMONIO
}

[Blackstone and the use by Bello in the formation of the Chilean Civil Code: An example of annulment of marriage]

\author{
JAVIER BarRientos GRANDON* \\ Academia Chilena de la Historia \\ Universidad Autónoma de Madrid, España**
}

\section{RESUMEN}

En el contexto de la circulación en Chile, durante la primera mitad del siglo XIX, de los Commentaries on the Laws of England de Blackstone, se examina la influencia que esta obra ejerció sobre Bello en la redacción de algunos artículos del primer borrador del libro I, De las personas, del Código Civil de Chile. En concreto, en sede de nulidad de matrimonio.

\section{Palabras Clave}

Andrés Bello - William Blackstone - Código Civil - Matrimonio.
AbSTRACT

In the context of the circulation in Chile, during the first half of the 19th century, of the Commentaries on the Laws of England by Blackstone, the influence that this work exerted on Bello in the writing of some articles of the first draft of the book I, De las personas, of the Civil Code of Chile, is examined. Specifically, in the nullity of marriage.

\section{KEYWORDS}

Andrés Bello - William Blackstone - Civil Code - Marriage.

ReCibido el 30 de abril de 2019 y ACEPTADo el 10 de junio de 2019

\section{INTRODUCCIÓN}

La prolongada estancia de Bello en Londres le dio ocasión, no sólo para consolidar su formación como jurista en general sino, muy particularmente, para ponerle en contacto con el common law que, a la sazón, contaba como una muy lograda

* Académico de número de la Academia Chilena de la Historia, profesor de Historia del Derecho y de las Instituciones en la Facultad de Derecho de la Universidad Autónoma de Madrid. Dirección postal: C/ Kelsen No 1, Ciudad Universitaria de Cantoblanco, 28049 Madrid - EsPaÑA. Dirección electrónica: javier.barrientos@uam.es

** Este estudio se ha realizado en el marco del Proyecto de I-D-I de la Dirección General de Investigación Científica y Técnica, Ref. Der2014-56291-C3-1-P. 
e influyente exposición: los Commentaries on the Laws of England de William Blackstone (1723-1780), maestro que había sido en Oxford de Jeremy Bentham (1748-1832), y a quien se debió una de sus más amplias y profundas críticas ${ }^{1}$.

Bello conoció en aquel tiempo los Commentaries de Blackstone, que llevó a Chile entre sus libros en la edición con notas de Joseph Chitty (1775-1841)2 La conservó en su biblioteca hasta el fin de sus días ${ }^{3}$, y la tuvo a la vista en la formación de sus Principios de derecho de jentes, publicados en Santiago de Chile en $1832^{4}$. Muchos años más tarde habría quien, incluso, llegaría a llamar a Bello como "el Blackstone del derecho civil americano"

No fue Bello, con todo, quien introdujo la obra de Blackstone en Chile. Consta que era un autor al que, aunque esporádicamente, se manejaba con anterioridad a su arribo al país en 1829, singularmente en sede de organización política. Así, por ejemplo, en 1822 era citado en el periódico El observador chileno, en un artículo rubricado "Naturalización”", cinco años después en El Telégrafo Mercantily Político en relación con la monarquía constitucional ${ }^{7}$, y en 1829 , diez días antes de que Bello tocara puerto en Valparaíso, José Joaquín de Mora recurría a Blackstone en uno de los artículos que incluía en El mercurio chileno en el que, bajo la rúbrica de "Preocupaciones forenses", mencionaba al jurista inglés a propósito del estudio de las reglas políticas y civiles ${ }^{8}$.

${ }^{1}$ Vide Posner, Richard A., Blackstone and Bentham, en The Journal of Law \& Economics, 19/3 (1976), pp. 569-606; Burns, J. H., Bentham and Blackstone: A Lifetime's dialectic, en Utilitas, 1/1 (1989), pp. 22-40; SCHOFIELD, Philip, The 'Least Repulsive' Work on a 'Repulsive Subject': Jeremy Bentham on William Blackstone's 'Commentaries on the Laws of England, en PAGE, Anthony y Prest, Wilfrid (eds.), Blackstone and His Critics (Oxford, Hart Publishing, 2018), pp. 23-40.

${ }^{2}$ Blackstone, William, Commentaries on the Laws of England [...] A new edition, with practical notes, by Joseph Chitty, Esq. Barrister at law (London, Printed for William Walker Strand, et alii, 1826), 4 vols.

${ }^{3}$ Ávila Martel, Alamiro de, Londres en la formación jurídica de Andrés Bello, en Revista de Estudios Histórico-Jurídicos, 7 (1982), p. 336; Velleman, Barry L., Andrés Bello y sus libros (Caracas, Casa de Bello, 1995), p. 134.

${ }^{4}$ B. [ello], A.[ndrés], Principios de derecho de jentes (Santiago de Chile, Imprenta de La Opinión, 1832), p. 6: "Las naciones modernas de Europa han reconocido el derecho de jentes como una parte de la jurisprudencia patria. Por aquellos estatutos [dice Sir W. Blackstone] que se han hecho de tiempo en tiempo en Inglaterra para reforzar esta lei universal y facilitar su ejecucion, no se han introducido reglas nuevas, sino solo se han declarado y esplicado las antiguas constituciones fundamentales del reino, que sin ellas dejaria de ser un miembro de la sociedad civilizada".

${ }^{5}$ Bosch, Ralph, Andres Bello, the Blackstone of American Civil Law, en American Bar Association Journal, 28 (1942), pp. 825, 852.

${ }^{6}$ El observador chileno, No 5 Santiago de Chile, octubre 28 de 1822, en Feliú Cruz, Guillermo, Colección de antiguos periódicos chilenos, 1822-1823 (Santiago de Chile, Biblioteca Nacional, 1962), pp. 148-149: "Aquellos que deseen cerciorarse a fondo sobre esta materia, por lo que respecta a las Leyes de Inglaterra, podrán consultar los Comentarios de Blackstone, Lib. I, Cap. X; Lib. II, Cap. XVI; y Lib. IV, Cap. XIX y XXXIII".

${ }^{7}$ En Sesiones de los Cuerpos Lejislativos de la República de Chile, 1811-1843 (Santiago de Chile, Imprenta Cervantes, 1891), XIV, p. 288, nota: "Por desgracia, tales máximas han sido sostenidas en el mundo por hombres eminentes que se han pronunciado por lo que la frase llama monarquía constitucional; se cuenta en este número el célebre Blackstone".

${ }^{8}$ El Mercurio chileno, № 15, Santiago de Chile, 15 de junio de 1829, "Preocupaciones forenses", pp. 706-707: "Blackstone, en su magnífica introduccion a los comentarios de las leyes 
En principio, aunque sabido que Bello recurrió a algunas obras de autores ingleses y también de los Estados Unidos de América, para formar algunos de los artículos del Código Civil, no es este un tema que, hasta ahora, haya ocupado la atención de la historiografía jurídica. En cierto modo, se mantiene culturalmente la general impresión que se forjó en su día Lira Urquieta: "La desperdigada legislación anglosajona no podía tener cabida: era demasiado extraña y diferente, no tanto en sus principios que derivaban del derecho romano, cuanto en sus manifestaciones externas. Algunos grandes autores de esa raza pudo citar Bello, como Blackstone y Kent, pero su aprovechamiento necesariamente fue escaso"'.

Ocasionalmente se ha señalado que algunas disposiciones del Código Civil chileno, aprobado en 1855, estarían influidas por los Commentaries de Blackstone. En general, la afirmación de esa influencia se ha basado en una nota que Miguel Luis Amunátegui incluyó en su edición del llamado "Proyecto inédito" del Código Civil. Se trata de un texto, escrito por Bello a propósito del Proyecto de Código Civil impreso en 1853, cuyo original manuscrito se conserva entre sus papeles ${ }^{10}$, y que Amunátegui incorporó como nota al artículo 234 de aquel proyecto, que versaba sobre la legitimación por el matrimonio subsecuente de los padres. En el lugar que aquí interesa se lee: "Siendo el fin del matrimonio, dice Blackstone, determinar una persona cierta en quien recaiga el cuidado, sustentacion i educacion de los hijos, es indudable que se consigue mejor este fin lejitimando solamente a la prole que nace de él i no a los hijos de las mismas personas habidos antes"11. De esta nota se ocupaba tempranamente Claro Solar ${ }^{12}$, y genéricamente se refería a ella Lira Urquieta para advertir acerca de la influencia de Blackstone en el Código Civil $^{13}$, y con remisión a éste también lo hacía de los Mozos ${ }^{14}$.

Se ha sugerido, igualmente, que en alguna regla o categoría admitida en el Código Civil podría hallarse el influjo de Blackstone sobre Bello. Tal es lo que, en su día, apuntó Guzmán Brito en relación con definición de “causa” que ofrece el inciso $2^{\circ}$ del artículo 1467 del Código Civil, en relación con la descripción que

inglesas, probó hasta la evidencia que no hai ninguna clase social a quien deba ser indiferente el estudio de las reglas que dirijen nuestras acciones civiles y políticas”. Otra referencia a Blackstone en el mismo artículo en p. 718.

${ }^{9}$ Lira Urquieta, Pedro, El Código Civil Chileno y su época (Santiago de Chile, Editorial Jurídica de Chile, 1956), p. 57.

${ }^{10}$ Archivo Central Andrés Bello, Colección manuscritos, 688 B446.

${ }^{11}$ Bello, Andrés, Obras completas de Don Andrés Bello, V. Proyecto de Código Civil (Santiago de Chile, Editorial Nascimento, 1932), III, p. 99. Que este texto fuera escrito en relación directa con el Proyecto de Código Civil de 1853 lo comprueba el que en un párrafo precedente se cita, precisamente, el artículo 230 de aquel Proyecto.

${ }^{12}$ Claro Solar, Luis, Explicaciones de Derecho Civil Chileno y Comparado. Las personas (Santiago de Chile, Establecimiento Poligráfico Roma, 1902), II, p. 337.

${ }^{13}$ Lira Urquieta, Pedro, Introducción, en Código Civil de la República de Chile (Caracas, Ministerio de Educación, 1954), I, p. XLIII: "[T]an pronto lo ha impresionado una ley de Las Partidas como un comentario de Blackstone [...]".

${ }^{14} \mathrm{De}$ Los Mozos, José Luis, Algunos aspectos de la influencia hispánica en el Código Civil de Andrés Bello, en Revista General de Legislación y Jurisprudencia, año 127 (1978), p. 458: “También hay referencia en sus notas a las leyes anglosajonas, comentadas por Blackstone y por Kent". 
hacía Blackstone de la consideration ${ }^{15}$. En el último tiempo, en fin, se ha llamado la atención sobre una influencia i n d i r e c t a del jurista inglés en algunas de las reglas sobre interpretación de la ley, y que habría operado a través del Code Civil de la Luisiana ${ }^{16}$.

En tal contexto, en este breve artículo, ofrezco una mínima información acerca de un caso concreto en el que consta que Bello recurrió a los Commentaries de Blackstone para fundar una regla asumida en el primer proyecto conocido del libro I, De las personas del Código Civil. Situada en sede de matrimonio, aunque ella finalmente no se consolidaría en el texto definitivo del Código, confirma que, efectivamente, Bello recurrió a la obra del jurista inglés en la época en la que preparaba el proyecto que se imprimiría en 1853.

\section{La obra de Blackstone en Chile en tiempos de la Formación DEL Código CIVIL}

Si la obra de Blackstone había circulado en Chile con anterioridad a la llegada de Bello, esa presencia se mantuvo en los años posteriores a su arribo al país. No sobrará recordar aquí que Mariano Egaña (1793-1846), tan ligado a Bello y a los primeros proyectos del Código Civil, tenía entre sus libros la edición de los Commentaries de Blackstone con las notas y adiciones de Edward Christian ${ }^{17}$.

Si en los primeros decenios del siglo XIX habían recurrido a los Commentaries, a propósito de instituciones políticas, autores que defendían el liberalismo, como José Joaquín de Mora, esa tendencia se mantuvo durante los decenios del siglo XIX en los que se trabajó en la formación de los proyectos del Código Civil.

Uno de los intelectuales que, por aquella época, recibió la influencia de las ideas de Blackstone fue Juan Bautista Alberdi (1810-1884). En los años que residió en Chile esa influencia, por ejemplo, se manifestaba ampliamente en sus trabajos sobre la "libertad de imprenta". De ella daban buena cuenta las frecuentes referencias que hacía al jurista inglés en su Lejislacion de la prensa en Chile, publicada en Valparaíso en $1846^{18}$. Unos años después, su compatriota Domingo Faustino Sarmiento (1811-1888) publicaba en Santiago sus Comentarios de la

\footnotetext{
${ }^{15}$ GuZmán Brito, Alejandro, La doctrina de la 'consideration' en Blackstone y sus relaciones con la 'causa' en el 'ius commune', en Revista de Estudios Histórico-Jurídicos, 25 (2003), p. 383, n. 28: "No puedo descartar que esta definición esté en la base de la definición de causa que ofrece el artículo 1.467 inciso $2^{\circ}$ del Código Civil de Chile: "Se entiende por causa el motivo que induce al acto o contrato"”.

${ }^{16}$ CAFFera, Gerardo, Interpretación de la Ley en los Códigos Civiles: Literalismo Sudamericano y Blackstone, en Latin American Legal Studies, 1 (2017), pp. 1-50. Sobre la influencia de Blackstone en las reglas del Code Civil de la Luisiana en esta sede ya había llamado la atención GuZMÁn BRITO, Alejandro, Las fuentes de las normas sobre interpretación de las leyes del 'Digeste des lois civiles (code civil)' de la Luisiana (1808/1825), en Revista de Estudios Histórico-Jurídicos, 31 (2009), pp. 171-195.

${ }^{17}$ Salinas Araneda, Carlos, La biblioteca de don Mariano Egaña, con especial referencia a sus libros de derecho, en Revista de Estudios Histórico-Jurídicos, 7 (1982), p. 523.

${ }^{18}$ Alberdi, Juan Bautista, Lejislacion de la prensa en Chile, o sea Manual del escritor, del impresor y del jurado (Valparaíso, Imprenta del Mercurio, 1846), pp. 23, 34, 40, 46, 51, 53, 54.
} 
Constitución de la Confederacion Arjentina, y en ellos también se apreciaba el peso de las opiniones de Blackstone, entre otras sedes, en la tocante a la libertad personal ${ }^{19}$. El influjo de Blackstone se mantendría en la segunda mitad del siglo XIX chileno, sobre todo, en los trabajos de algunos autores dedicados al estudio del derecho constitucional, tales como Manuel Carrasco Albano (1834-1873) ${ }^{20}$ y José Victorino Lastarria $(1817-1888)^{21}$.

En este tiempo las opiniones de Blackstone también comenzaron a invocarse en la discusión de ciertas cuestiones que sobrepasaban los límites de las instituciones políticas, y que tocaban a algunas que interesaban a materias que caían dentro de la esfera de las reglas que se entendía habían de tener un sitio en el Código Civil. Tal fue, por ejemplo, la discusión que, con ocasión de la reforma del "Reglamento de elecciones", se suscitó en 1842 entre Bello y su discípulo Manuel Antonio Tocornal (1817-1867) sobre la derogación de las leyes políticas. Este último publicó en El semanario de Santiago cinco artículos para defender su opinión, en contra de la que profesaba Bello en las páginas de El Araucano. En el que apareció en la edición de 27 de octubre de 1842 Tocornal incluía el siguiente párrafo: "La lei posterior concebida en términos negativos, ha dicho Blackstone, autoridad respetable, deroga la anterior, aun cuando no lo esprese; porque la negacion envuelve virtualmente la derogacion" 22 . Aunque ligada a una cuestión política, esta discusión recaía sobre un punto central en sede de vigencia de la ley, del que se ocupaba por aquella época Bello en los primeros borradores del título preliminar. Si bien no es este lugar para tratar de la opinión de Amunátegui que se va a recordar ahora, éste escribía en la biografía de Tocornal que publicara en 1894 que: "Esta polémica fue una de las causas que influyeron para que se pusiera el adverbio jamás en el inciso $1^{\circ}$ del artículo 9 del Código Civil Chileno"23.

En el mismo espacio de cuestiones ligadas al derecho civil, también la obra de Blackstone tuvo algún uso forense, sobre todo en ciertos procesos en los que, por la presencia de ingleses en Chile, era necesario determinar cuál era el derecho inglés aplicable en la solución del pleito. Así, en un "Parecer. Sobre el testamento de un inglés otorgado en Arequipa”, redactado por el abogado Manuel Carvallo (1808-1867), y fechado en Santiago el 7 de abril de 1837, se recurría a Blackstone para justificar la siguiente afirmación: "Un testamento firmado o sellado por el testador, y publicado a presencia de testigos, tiene, según las leyes inglesas, todos los requisitos para transmitir a los legatarios y herederos voluntarios no solo la propiedad personal, sino también la propiedad real del testador" ${ }^{24}$. La remision

19 Sarmiento, Domingo Faustino, Comentarios de la Constitución de la Confederacion Arjentina, con numerosos documentos ilustrativos del texto (Santiago de Chile, Imprenta de Julio Belin i Cia., 1853), pp. 187, 202.

${ }^{20}$ Carrasco Albano, Manuel, Comentarios sobre la Constitución Política de 1833 (Valparaíso, Imprenta del Mercurio, 1858), p. 112.

${ }^{21}$ Lastarria, José Victorino, Lecciones de politica positiva. Profesadas en la Academia de Bellas Letras (Santiago de Chile, Imprenta del Ferrocarril, 1874), pp. 270, 287.

${ }^{22}$ El Semanario de Santiago, No 17, Octubre 27 de 1842, p. 140.

${ }^{23}$ AmunÁtegui, Miguel Luis, Ensayos biográficos (Santiago de Chile, Imprenta Nacional, 1894), III, p. 23.

${ }^{24}$ Carvallo, Manuel, "Parecer. Sobre el testamento de un inglés otorgado en Arequipa”, 
a los Commentaries era la siguiente: "Blackstone, Comentario a las leyes de Inglaterra, Lib. 2, cap. 32"25.

Las ligeras observaciones precedentes permiten acercarse, de algún modo, al contexto de la cultura jurídica chilena en tiempos de la formación del Código Civil en cuanto a la circulación y uso de la obra de Blackstone en el país. Ellas, así, muestran que no era sólo peculiar a Bello el recurso a los Commentaries, y que éste no se limitaba a temas de índole política.

\section{El MATRIMONIO Y SU NULIDAD: LA INFLUENCIA DE BLACKSTONE}

Bello durante su estancia en Inglaterra se formó una muy favorable opinión acerca de las leyes y costumbres de aquel país en cuanto al matrimonio y las relaciones de familia. De aquel buen concepto dejó más de un testimonio a propósito de ciertos artículos del Código Civil.

En una nota puesta al artículo $5^{\circ}$ del "Título VIII. De las asignaciones forzosas", publicado en El Araucano de 27 de mayo de 1842, en la que recordaba la discusión que había en la Comisión acerca de las legítimas y de la libertad de testar, escribía: "En este Proyecto no se impone al padre la obligacion de emplear cierta cantidad de sus bienes, fuera de las lejítimas, en beneficiar a uno o más de sus descendientes a su arbitrio, dividiéndola entre ellos como quiera. Se le autoriza, pues, para disponer libremente de la mitad de sus bienes, i se suprime la mejora del tercio, invencion peculiar de los godos. Esta supresion es una de las reformas en que tenemos mas diverjencia de opiniones. La creemos, con todo, apoyada en razones de gran peso. En el corazon de los padres tiene el interés de los hijos una garantia mucho mas eficaz que la proteccion de la lei; i el beneficio que deben estos alguna vez a la intervencion del lejislador, es mas que contrapesado por la relajacion de la disciplina doméstica, consecuencia necesaria del derecho perfecto de los hijos sobre casi todos los bienes del padre. Así vemos que ni aun las lejítimas fueron conocidas en Roma, mientras a la sombra de las virtudes republicanas se mantuvieron puras las costumbres i severa la disciplina doméstica. Las lejítimas no son conocidas en la mayor parte de la Gran Bretaña i de los Estados Unidos de América; i tal vez no hai países donde sean mas afectuosas i tiernas las relaciones de familia, mas santo el hogar domestico, mas respetados los padres, o procurados con mas ansia la educacion i establecimiento de los hijos. Cuando mas suave el yugo de las leyes, mas poderosa es menester que sea la venerable judicatura que la naturaleza confiere a los padres" ${ }^{26}$. Una opinión similar vertía en la citada nota puesta por Amunátegui al artículo 234 del denominado "Proyecto inédito", en la que se lee la siguiente observación de Bello: "La Inglaterra no se dejó arrastrar por la influencia del derecho canónico, aun cuando todavía era una de las naciones más obsecuentes y sumisas a la Iglesia [...] Según las leyes de ese país, que es uno de los más notables por la ternura de los afectos de familia y la disciplina

en Gaceta de los Tribunales, 16 de abril de 1842, № 19, p. 73.

${ }^{25}$ Ibíd.

${ }^{26}$ Bello, Andrés, Obras completas de Don Andrés Bello, III. Proyecto de Código Civil (Santiago de Chile, Editorial Nascimento, 1932), I, p. 94. 
doméstica, no hay legitimacion posible para los hijos ilejítimos, sino la del artículo 230 del Proyecto" 27.

Si su experiencia personal, y el largo tiempo que vivió en la sociedad inglesa, hubieron de pesar en el juicio que Bello se forjó acerca de sus costumbres y leyes en relación con el matrimonio y la familia, con seguridad la lectura de las secciones pertinentes de los Commentaries de Blackstone también tuvieron que incidir en esa general buena opinión, enmarcada, en todo caso, en las concepciones liberales más amplias de que se hizo partícipe en Londres. No resulta extraño, así, que cuando emprendió la tarea de redactar los artículos del Código Civil tocantes a las personas y sus relaciones de familia acudiera a la obra del jurista inglés.

Bello, con posterioridad al año 1847, que fue aquel en que hizo imprimir una versión revisada del Libro de los contratos i obligaciones convencionales, hubo de comenzar a trabajar en el proyecto de Libro I, De las personas, del que habría de ser el libro respectivo del Proyecto de Código Civil impreso en 1853. La primera versión de este libro I se conserva como un borrador manuscrito entre los papeles de Bello, ya bajo el título De las personas, dividido, al menos, en veinte títulos, con numeración independiente para sus artículos, y varios de ellos acompañados de notas, explicativas en algún caso, o remisivas a fuentes en otros. Sobre la base de un texto inicial, escrito en limpio con letra de amanuense, hay numerosas correcciones y anotación de puño y letra de Bello ${ }^{28}$.

En ese borrador manuscrito se lee la versión más antigua conocida del "Título 4. Del matrimonio". Escrito con letra de amanuense, consta de veinte artículos, numerados correlativamente, en arábigos, desde el 1 en adelante. Sólo en dos de ellos hay anotaciones, que remiten a fuentes, escritas entre paréntesis al final de su texto y con letra del mismo amanuense. Sobre esta versión manuscrita trabajó Bello y, como era habitual, constan de su puño y letra las correcciones, cambios de redacción, eliminación de incisos o de artículos mediante el tarjado de su texto, así como la inclusión en los márgenes de los nuevos textos. Su labor sobre la versión del amanuense fue menos profunda que la que había realizado sobre la versión original de otros títulos, por ejemplo, del título II. Tarjó el contenido del artículo 2, y lo incluyó en el artículo 3, tarjó también el artículo 15, y al artículo 17 le dio una nueva redacción. Modificó la redacción de los demás artículos, salvo en los casos del 1 y 6 , y a algunos de ellos les agregó notas explicativas o remisivas a fuentes. Como resultado de todas esas innovaciones, en el borrador manuscrito se puede reconstruir una versión final de este título IV. Ella mantenía su número de 20 artículos, porque si bien había eliminado dos artículos, incorporó dos nuevos.

En el citado borrador manuscrito del referido "Título 4. Del matrimonio", en la versión escrita por amanuense, se leía un artículo, el 15, acompañado por una remisión expresa a Blacsktone en un paréntesis final: "Despues de la muerte de uno de los consortes, no se podrá impugnar la validez del matrimonio que se haya

\footnotetext{
${ }^{27}$ Bello, Andrés, Obras completas, cit. (n. 11), III, p. 99.

${ }^{28}$ Archivo Central Andrés Bello, Colección manuscritos, 684 B446, fol. 16r-21v.
} 
contraido con las solemnidades requeridas por la lei, aunque el matrimonio haya sido verdaderamente nulo por algun impedimento legal. (Blacstone)"29.

Bello, ya en aquel momento del proceso codificador, había asumido el principio conforme al cual se confiaba a la autoridad eclesiástica la decisión acerca de la validez del matrimonio. Sin embargo, no lo llevaba hasta sus últimas consecuencias, pues incluía algunas reglas acerca de esa materia. Así ya lo dejaba ver el modo a través del cual reconocía dicho principio en el artículo 2 del expresado borrador: "[inc. $1^{\circ}$ ] No pueden contraer matrimonio los varones que no hayan cumplido diez $i$ seis años, ni las mujeres que no hayan cumplido catorce. El Gobierno, sin embargo, podrá conceder dispensa de edad por motivos graves. [inc. 20] La infraccion de esta lei será castigada con la pena que el código criminal prescriba, pero sobre la validez del matrimonio decidirá la autoridad eclesiástica"30.

Este artículo no iba acompañado de ninguna referencia en el borrador manuscrito, pero es muy probable que su inspiración la hubiera hallado Bello en la lectura de Blackstone, pues refleja con mucha similitud las ideas expresadas por el jurista inglés en el lugar correspondiente de sus Commentaries: "Our law considers marriage in no other light than as a civil contract. The holiness of the matrimonial state is left entirely to the ecclesiastical law: the temporal courts not having jurisdiction to consider unlawful marriage as a sin, but merely as a civil inconvenience. The punishment, therefore, or annulling, of incestuous or other unscriptural marriages, is the province of the spiritual courts; which act pro salute anima (for the welfare of the soul). And, taking it in this civil light the law treats it as it does all other contracts" 31 .

Bello acompañó en el borrador a este artículo de una nota, que tiende a confirmar que pesó en él la lectura del pasaje de los Commentaries de Blackstone que se ha transcrito. Esa nota la reprodujo, con un breve agregado, en el artículo 118 del Proyecto de Código Civil impreso en 1853. En ella advertía que: "Muchos encontrarán aquí un vacío, i querrían que se expusieran a la larga los impedimentos para contraer matrimonio. Pero ‘a qué poner como leyes las que no dirijirian a la autoridad eclesiástica, única competente en materia de martrimonios? [...] En este orden de cosas, la lei civil no puede menos de estar al juicio de la autoridad eclesiastica, sobre la validez del matrimonio. Todo lo que puede hacer el poder temporal es reprobar y sujetar a una pena el matrimonio permitido por la autoridad eclesiástica, o negar a ese matrimonio los efectos civiles, cuando lo crea de perniciosas consecuencias para la moral doméstica"32.

En esa línea se entendía, también, que en el inciso $1^{\circ}$ del artículo 3 se expresaran, en términos generales, los requisitos para la validez del matrimonio, y que en su inciso $2^{\circ}$ se reiterara la regla de la parte final del artículo 2: "[inc. $1^{\circ}$ ] Para la validez del matrimonio se requiere, ademas, $1^{\circ}$. El consentimiento de los contrayentes.

\footnotetext{
${ }^{29}$ Ibíd., fol. 20r.

${ }^{30}$ Ibíd.

${ }^{31}$ Blackstone, William, Commentaries on the Laws of England, cit. (n. 2), I, cap. XV, $₫$ 583 [434].

${ }^{32}$ Archivo Central Andrés Bello, Colección manuscritos, 684 B446, fol. 16v; cfr. BeLlo, Andrés, Obras completas de Don Andrés Bello, XII, Proyecto de Código Civil (1853), (Santiago de Chile, Impreso por Pedro G. Ramírez, 1888), p. 36.
} 
$2^{\circ}$. Que no haya impedimento dirimente para la union de los contrayentes; o que de la autoridad competente se haya obtenido dispensa de los impedimentos de esta clase. $3^{\circ}$. Que el matrimonio se contraiga ante competente sacerdote $i$ a presencia de dos testigos a lo menos. [inc. 2०] Toca a la autoridad eclesiástica decidir sobre la validez del matrimonio que se ha de contraer o se ha contraido"33.

Este artículo, que tampoco iba acompañado de remisión alguna, pareciera también influido por la lectura de la obra de Blackstone, en la que se exponían, precisamente desde la perspectiva de la ley civil, las tres exigencias para la validez del matrimonio: "And, taking it in this civil light the law treats it as it does all other contracts: allowing it to be good and valid in all cases, where the parties at the time of making it where, in the first place, willing to contract; secondly, able to contract; and, lastly, actually did contract, in the proper forms and solemnities requiried by law" 34 .

Algo más allá iba el artículo 15 que queda transcrito, y que es aquel que concluía con la remisión a Blackstone. Precisamente en la referencia al jurista inglés pareciera hallarse la decisión de Bello de incluir aquella regla que impedía impugnar la validez del matrimonio que se hubiera contraído con las solemnidades requeridas por la ley, aunque el matrimonio hubiera sido verdaderamente nulo por algún impedimento legal.

La señalada remisión, que consta en el artículo 15 en el borrador manuscrito de este título, comprueba que Bello recurrió a los Commentaries, al menos en este campo, para redactar el que sería el Proyecto de Código Civil impreso en 1853. A pesar de lo genérica de la referencia, si se va al lugar correspondiente de los Commentaries se puede comprobar la exactitud de la indicación hecha al final de este artículo: "And no marriage is voidable by the ecclesiastical law, after the death of either of the parties, nor during their lives, unless for de canonical impediments of pre-contract, if that indeed still exists; of consanguinity; and of affinity; or corporal imbecility; subsisting previous to the marriage" ${ }^{35}$. ["Y ningún matrimonio es anulable por la ley eclesiástica, después de la muerte de cualquiera de las partes, ni durante sus vidas, a menos que se trate de impedimentos canónicos anteriores al contrato, si es que todavía existen; de consanguinidad; y de afinidad; o imbecilidad corporal; si existían antes del matrimonio"].

La correspondencia de contenido entre lo expuesto por Blackstone y la regla asumida por Bello en aquel originario artículo 15 del borrador manuscrito es evidente. Es ella, también, la que da pie para sugerir que en el codificador chileno también pesó la lectura de los Commentaries en la concepción originaria de la regla que confiaba a la autoridad civil la decisión acerca de la validez del matrimonio.

Bello, durante la revisión que hizo de ese primer borrador, cambió de criterio en cuanto a las reglas que había de contener el Código Civil en relación con la validez del matrimonio. Si ya en el momento inicial había explicado que carecía se sentido incluir una detallada regulación de los impedimentos, luego extendió esa idea al campo de la mención de los requisitos de validez del matrimonio, que

${ }^{33}$ Ibíd., fol. 20r-20v.

${ }^{34}$ Blackstone, William, Commentaries on the Laws of England, cit. (n. 2), I, cap. XV, $\$$ 583 [434].

${ }^{35}$ Ibíd., chap. XV, $\$ 592$, [440]. 
había hecho en el inciso $1^{\circ}$ del artículo $3^{\circ}$ que, así, resultó eliminado y, por ende, no incluido en la versión impresa del Proyecto en 1853. Bello justificaba esta decisión en una observación manuscrita, al margen del artículo 4 del borrador. En la versión final trasladó a este artículo parte del contenido del originario artículo 3 , y por ello en la nota lo explicaba como consecuencia del reconocimiento de la jurisdicción eclesiástica en la materia, salvo en lo relativo a la enumeración general de los requisitos del matrimonio que se contenían en la definición del matrimonio, puesta en su artículo 1. Esta nota, que no pasó al Proyecto impreso en 1853 decía: "En realidad, como el artículo precedente pudiera pasarse a un único en que se reconociese la competencia de la autoridad ecc.a en esta materia, excepto en cuanto las principales que para el contrato matrimonial explica la definicion del artículo $1^{\text {"”36. }}$.

En armonía con este nuevo criterio ha de situarse la decisión de Bello de eliminar el referido artículo 15 del borrador, que tarjó completamente y, en consecuencia, tampoco se recibió en el Proyecto de 1853.

Esta decisión de Bello oculta la influencia ejercida por Blackstone en el primer borrador de libro I del Código Civil en sede de nulidad matrimonial.

\section{CONClusión}

El breve examen del artículo 15 del primer borrador del "Título IV. Del matrimonio" del libro I del Código Civil, y de la remisión final que Bello hacía a Blackstone, permite comprobar que en sede de nulidad de matrimonio Bello tuvo presente la obra del jurista inglés.

Esa influencia es singularmente importante, pues, se produce respecto del principio cardinal sobre el cual se estructuró la disciplina de la validez y nulidad del matrimonio, a saber, la de confiar a la autoridad eclesiástica la decisión acerca de la validez del matrimonio.

Permite, en fin, ofrecer nuevos datos acerca de la circulación de la obra de Blackstone en Chile en tiempos de la formación del Código Civil, y del peso que tuvo la tradición jurídica inglesa en ella.

\section{BIBLIOGRAFÍA}

Alberdi, Juan Bautista, Lejislacion de la prensa en Chile, o sea Manual del escritor, del impresor y del jurado (Valparaíso, Imprenta del Mercurio, 1846).

AmunÁtegui, Miguel Luis, Ensayos biográficos (Santiago de Chile, Imprenta Nacional, 1894), III.

Ávila Martel, Alamiro de, Londres en la formación jurídica de Andrés Bello, en Revista de Estudios Histórico-Jurídicos, 7 (1982), pp. 317-350.

B. [ello], A.[ndrés], Principios de derecho de jentes (Santiago de Chile, Imprenta de La Opinión, 1832).

Bello, Andrés, Obras completas de Don Andrés Bello, XII, Proyecto de Código Civil (1853), (Santiago de Chile, Impreso por Pedro G. Ramírez, 1888).

${ }^{36}$ Archivo Central Andrés Bello, Colección manuscritos, 684 B446, fol. 16v. 
Bello, Andrés, Obras completas de Don Andrés Bello, V. Proyecto de Código Civil (Santiago de Chile, Editorial Nascimento, 1932), III.

Bello, Andrés, Obras completas de Don Andrés Bello, III. Proyecto de Código Civil (Santiago de Chile, Editorial Nascimento, 1932), I.

Blackstone, William, Commentaries on the Laws of England [...] A new edition, with practical notes, by Joseph Chitty, Esq. Barrister at law (London, Printed for William Walker Strand, et alii, 1826), 4 vols.

Bosch, Ralph, Andres Bello, the Blackstone of American Civil Law, en American Bar Association Journal, 28 (1942), pp. 825-852.

Burns, J. H., Bentham and Blackstone: A Lifetime's dialectic, en Utilitas, 1/1 (1989), pp. 22-40.

CAFFERA, Gerardo, Interpretación de la Ley en los Códigos Civiles: Literalismo Sudamericano y Blackstone, en Latin American Legal Studies, 1 (2017), pp. 1-50.

Carrasco Albano, Manuel, Comentarios sobre la Constitución Politica de 1833 (Valparaíso, Imprenta del Mercurio, 1858).

Carvallo, Manuel, Parecer. Sobre el testamento de un inglés otorgado en Arequipa, en Gaceta de los Tribunales, 16 de abril de 1842, № 19.

Claro Solar, Luis, Explicaciones de Derecho Civil Chileno y Comparado. Las personas (Santiago de Chile, Establecimiento Poligráfico Roma, 1902), II.

De los Mozos, José Luis, Algunos aspectos de la influencia hispánica en el Código Civil de Andrés Bello, en Revista General de Legislación y Jurisprudencia, año 127 (1978).

Feliú Cruz, Guillermo, Colección de antiguos periódicos chilenos, 1822-1823 (Santiago de Chile, Biblioteca Nacional, 1962).

GuZMÁn BRITO, Alejandro, La doctrina de la 'consideration' en Blackstone y sus relaciones con la 'causa' en el 'ius commune', en Revista de Estudios Histórico-Jurídicos, 25 (2003), pp. 375-406.

GUZMÁN BRITO, Alejandro, Las fuentes de las normas sobre interpretación de las leyes del 'Digeste des lois civiles (code civil)' de la Luisiana (1808/1825), en Revista de Estudios Histórico-Jurídicos, 31 (2009), pp. 171-195.

Lastarria, José Victorino, Lecciones de politica positiva. Profesadas en la Academia de Bellas Letras (Santiago de Chile, Imprenta del Ferrocarril, 1874).

Lira Urquieta, Pedro, Introducción, en Código Civil de la República de Chile (Caracas, Ministerio de Educación, 1954), I.

El Código Civil Chileno y su época (Santiago de Chile, Editorial Jurídica de Chile, 1956).

Posner, Richard A., Blackstone and Bentham, en The Journal of Law \& Economics, 19/3 (1976), pp. 569-606.

Salinas Araneda, Carlos, La biblioteca de don Mariano Egaña, con especial referencia a sus libros de derecho, en Revista de Estudios Histórico-Jurídicos, 7 (1982), pp. 389-540.

Sarmiento, Domingo Faustino, Comentarios de la Constitución de la Confederacion Arjentina, con numerosos documentos ilustrativos del texto (Santiago de Chile, Imprenta de Julio Belin i Cia., 1853).

SCHOFIELD, Philip, The 'Least Repulsive' Work on a 'Repulsive Subject': Jeremy Bentham on William Blackstone's 'Commentaries on the Laws of England, en PAGE, Anthony y Prest, Wilfrid (eds.), Blackstone and His Critics (Oxford, Hart Publishing, 2018), pp. 23-40.

Sesiones de los Cuerpos Lejislativos de la República de Chile, 1811-1843 (Santiago de Chile, Imprenta Cervantes, 1891), XIV.

Velleman, Barry L., Andrés Bello y sus libros (Caracas, Casa de Bello, 1995). 